\title{
Secondary Parathyroid Hormone Resistance
}

National Cancer Institute

\section{Source}

National Cancer Institute. Secondary Parathyroid Hormone Resistance. NCI Thesaurus.

Code C131132.

Parathyroid hormone (PTH) resistance caused by vitamin D deficiency and characterized by clinically increased PTH concentrations with relative hyperphosphatemia. The diagnosis can be confirmed by finding of a normal cyclic adenosine monophosphate (CAMP) response to PTH infusion, but deficient phosphaturic response, indicating a defect distal to CAMP generation in renal cells. 